\title{
INJECTION ACCELERATION AND EXTRACTION OF HIGH INTENSITY PROTON BEAM FOR THE "NEUTRINO FACILITY" PROJECT AT BNL *
}

\author{
N. Tsoupas, J. Alessi, D. Barton, G. Ganetis, A. Jain, Y. Lee, I. Marneris, W. Meng, D. Raparia, \\ T. Roser, A. Ruggiero, J. Tuozzolo, P. Wanderer, B. Weng, BNL, Upton, NY 11973 USA
}

\begin{abstract}
The proposed "neutrino-production" project[1,2] to be built at the Brookhaven National Laboratory (BNL) requires that the neutrino-production target be bombarded by a high intensity proton beam-pulse of $\sim 90 \times 10^{12}$ protons of $28 \mathrm{GeV}$ in energy and at a rate of $2.5 \mathrm{~Hz}$, resulting in a $1 \mathrm{MW}$ power of proton beam deposited on the target for the production of the neutrinos. In this paper we investigate the possibility of producing this high intensity proton beam, using as the main accelerator the Alternating Gradient Synchrotron (AGS) at the Brookhaven National Laboratory (BNL).

The following aspects of the project are reported in this paper: a) The beam injection into the AGS synchrotron of $1.2 \mathrm{GeV} \mathrm{H}^{-}$beam produced by a super-conducting LINAC[3]; b) The effect of the eddy currents induced on the vacuum chamber of the circulating beam during the "ramping" of the main magnets of the AGS. c) The method of the beam extraction from the AGS and the optics of the $28 \mathrm{GeV}$ beam extracted from the AGS.
\end{abstract}

\section{H' BEAM INJECTION INTO AGS}

The AGS synchrotron was operating in early years [5] with protons being injected from a $200 \mathrm{MeV} \mathrm{H}^{-}$beam produced by a LINAC. In this section we discuss the proposed injection into the $\mathrm{AGS}$, of an $\mathrm{H}^{-}$beam produced by a $1.2 \mathrm{GeV}$ Supercoducting LINAC, (SC_Linac).

\subsection{Layout of $H$ - Injection region in $A G S$}

Figure 1 is a schematic diagram of the accelerators system involved in the production of the $28 \mathrm{GeV}$ proton beam. Namely the $200 \mathrm{MeVLinac}$, the SC_linac, and the AGS. The $\mathrm{H}^{-}$injection region has been chosen to be the location of the B20 straight section of the AGS. A schematic diagram of the injection region is shown in Fig. 2. In this diagram the following components of the injection region are shown:

a) Three of the main magnets $(\mathrm{B} 19, \mathrm{~B} 20, \mathrm{C} 01)$ of the AGS.

b) The stripping foil, shown inside the magnet $\mathrm{C} 01$.

c) The closed beam orbits which is "locally-bumped" during the time period for $\mathrm{H}^{-}$beam injection [2].

d) The closed orbit of the circulating proton-beam with no beam bumps (labeled as "Circulating p-beam" in Fig. 2 ) e) The trajectory of the injected $\mathrm{H}^{-}$beam. The direction of the injected $\mathrm{H}^{-}$is defined by the coordinate point $\left(\mathrm{x}, \mathrm{x}^{\prime}\right)$ shown in Fig. 2 [2]. The coordinate $\mathrm{x}$ is the distance from the center point of the straight section SS_B19 and X' is the angle between the direction of the central particle of

* Work Performed under Contract No. DE-AC02-98CH10886 with the U.S. Department of Energy. the $\mathrm{H}^{-}$beam and the direction of the straight SS_B19.

f)The trajectories of the $\mathrm{H}^{-}$beam which is not stripped by the stripping foil. The trajectory of the partially stripped $\mathrm{H}^{-}$beam $\left(\mathrm{H}^{0}\right)$ and the trajectories of the electrons (e) emanating from the stripping foil. All these particles must be collected downstream of the stripping foil into various "beam-dumps".

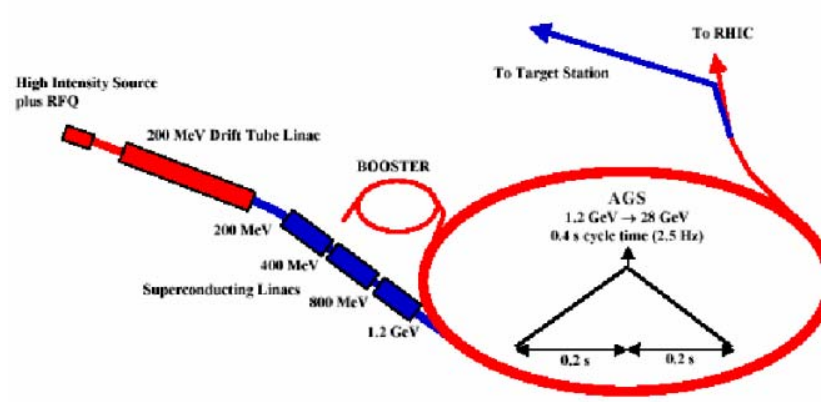

Figure 1. Schematic diagram of the accelerators for the "neutrino production".

\subsection{Generating the "local beam" bumps for the H-beam injection.}

In order to make the circulating proton beam collinear with the $\mathrm{H}^{-}$injected beam at the injection point ("stripping foil" in Fig. 2), the circulating proton beam is "locally bumped" by using two horizontal "bump-magnets" [2].

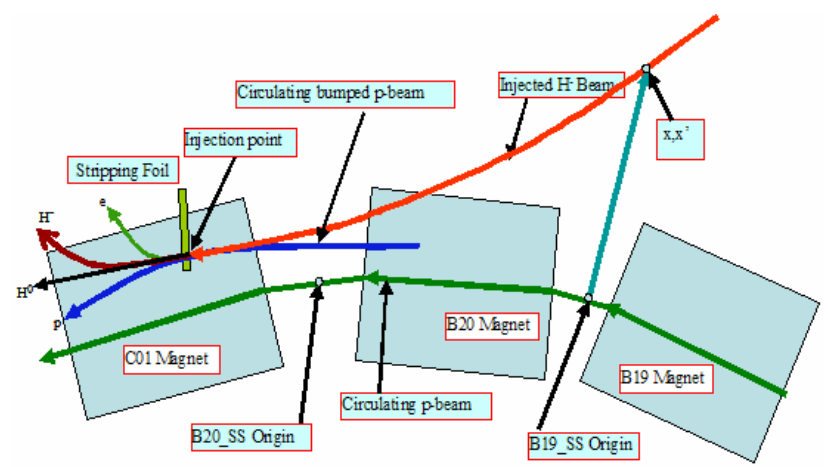

Figure 2. Schematic diagram of injection region in AGS.

Figure 3 plots the closed beam orbit in AGS with the "local bump" turned OFF (red line) and turned ON (blue line). Figure 4 shows a section of the bumped orbit of the beam at the location of the C01 main-magnet (see fig. 2) The maximum displacement of the closed orbit $(\sim 56 \mathrm{~mm})$ occurs at the center of the 75 inches long $\mathrm{C} 01$ magnet. 


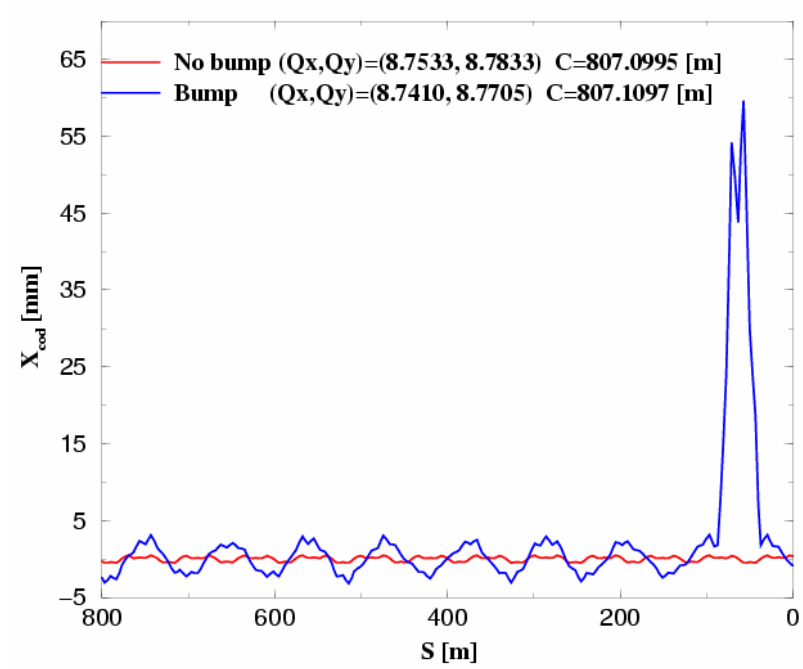

Figure 3: Closed orbits in the AGS. The red and blue curves corresponds to bumps OFF, and $\mathrm{ON}$

Each of the other lines shown in Fig. 4, are trajectories of the $\mathrm{H}^{-}$injected beam corresponding to a different $\left(\mathrm{x}, \mathrm{x}^{\prime}\right)$ location and direction of the $\mathrm{H}^{-}$injected beam. The values of ( $\mathrm{x} \mathrm{x}$ ') that were used to generate the trajectories shown in figure 4 are shown also on the same figure 4. Each of the $\mathrm{H}^{-}$trajectories is tangent to the "bumped-closed orbit" (black line) and this has been achieved by adjusting the initial conditions $\left(\mathrm{x}, \mathrm{x}^{\prime}\right)$ of the $\mathrm{H}^{-}$injected beam.

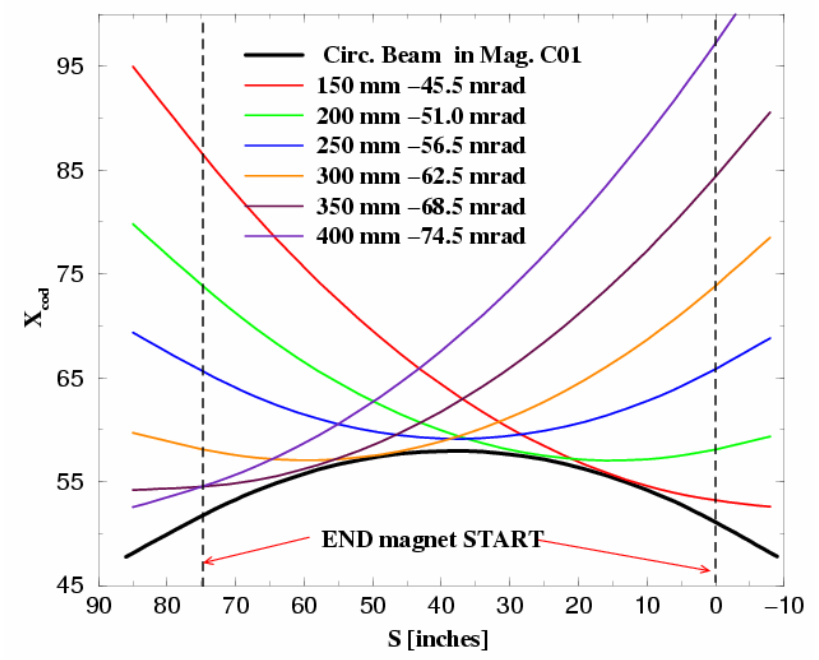

Figure 4. A section of the "Bumped Closed Orbit" (black line) inside the C01 magnet of AGS.

From all the possible $\mathrm{H}^{-}$trajectories shown in Fig. 4, the $\mathrm{H}^{-}$trajectory with $\left(\mathrm{x}, \mathrm{x}^{\prime}\right)=(250 \mathrm{~mm},-56.5 \mathrm{mrad})$ has been selected as the "injection trajectory" (blue line in Fig 4).

The various $\mathrm{H}^{-}$trajectories shown in figure 4 have been calculated using the code AGS_BATE[4].

In addition to the "local-bump" of the closed orbit discussed above, a set of two fast time varying bumps (horizontal and vertical) of the closed orbit may also be superimposed to help optimize the "beam painting" of the AGS phase space.

\subsection{Calculations of the beam parameters at the injection point of the $H^{-}$beam.}

In order to minimize the emittance blow-up of the $\mathrm{H}^{-}$ injected beam into the AGS, the beam parameters of the injected $\mathrm{H}^{-}$beam must match those of the circulating beam in AGS at the "injection point" (Fig. 2). In this write-up the "injection-point" is the point where the $\mathrm{H}^{-}$ injected beam and the circulating beam intersect the plane of the stripping foil. The beam parameters of the circulating beam in AGS at the "injection point" have been calculated, for an injection beam energy of $1.2 \mathrm{GeV}$, using the computer code AGS_BATE[4]. The calculated beam parameters appear in Table 1 . The proximity of the $\mathrm{H}^{-}$injection line to the AGS main magnets requires that the fringe field of the AGS magnet is taken into account for the calculations of the $\mathrm{H}^{-}$trajectories and the beam parameters at the injection points. In order make the matching calculations easier, we have selected another point "Matching point" (point $\mathrm{x}, \mathrm{x}$ ' in Fig. 2). The beam parameters at this point were calculated using the beam parameters at the "Injection point" and the first order transfer Matrix (R) between matching and injection point. The R-matrix was calculated by tracing the $\mathrm{H}^{-}$beam in the fringe field of the B20 and C01 main magnets of AGS.

TABLE 1: The beam parameters at the "Injection point" and "Matching point" (see text).

\begin{tabular}{|l|c|c|c|c|c|c|}
\hline Loc. & $\beta_{\mathrm{x}}[\mathrm{m}]$ & $\alpha_{\mathrm{x}}$ & $\eta_{\mathrm{x}}[\mathrm{m}]$ & $\eta_{\mathrm{x}}^{\prime}$ & $\beta_{\mathrm{y}}[\mathrm{m}]$ & $\alpha_{\mathrm{y}}$ \\
\hline INJ. & 28.0 & -1.00 & -1.25 & -0.05 & 8.00 & 0.43 \\
\hline MAT. & 26.6 & -1.32 & -1.00 & -0.10 & 16.10 & 1.75 \\
\hline
\end{tabular}

\subsection{Handling of the $H^{-} H^{0}$ and $e^{-}$beams.}

The $\mathrm{H}^{-}$injected beam will not be stripped totally of its electrons by the stripping foil, therefore the emerging beam from the foil will also consists of: $\mathrm{H}^{-}$ions, $\mathrm{H}^{0}$ particles and electrons (e). Except for the fully stripped $\mathrm{H}^{-}$ (protons) the rest of the particles must be "dumped" into a material which can absorb the deposited energy. The different rigidity of these three particles species, may require specially designed beam dumps[5].

\section{ACCELARATION OF PROTONS}

The proposed main-magnet cycle, of frequency $2.5 \mathrm{~Hz}$. for the "neutrino production" operations, requires a time interval of $\sim 90 \mathrm{msec}$ between the beam injection and extraction. This fast ramp rate of the AGS main magnets will generate eddy currents in the following materials: a) the coils that power the magnet, b) the $1.9 \mathrm{~mm}$ thick vacuum chamber, and c) the iron of the main magnet. The eddy currents will generate Ohmic heating in the coils of the magnets, in the wall of the vacuum chamber, and in 
the iron of the magnet which consists of laminations 3.2 $\mathrm{mm}$ thick and held together by two $2.5 \mathrm{~cm}$ thick plates at each end of the magnet. The eddy currents in the wall of the vacuum chamber will also generate time varying magnetic multipoles at the region of the circulating beam. The Ohmic effects on the materials mentioned above and the multipoles generated by the eddy currents have been studied theoretically and appear in ref.[2]. Figure 5 is a plot of the measured temperature in the wall of the vacuum chamber, when the coils of one of the AGS magnets is powered at a frequency of $2.5 \mathrm{~Hz}$.

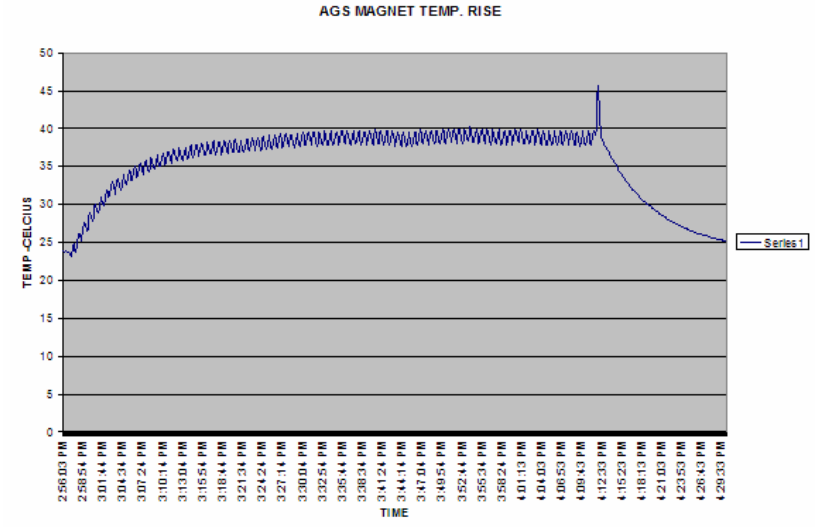

Figure 5. Temperature in the wall of the vacuum chamber as a function of time. The temperature levels at $\sim 40{ }^{\circ} \mathrm{C}$.

\section{BEAM EXTRACTION FROM AGS}

At present the AGS synchrotron is equipped with a Fast Beam Extraction (FEB) system[6] which is used to extract the circulating bunched beam in AGS to the AtR beam transfer line[7] for beam injection into RHIC[7] and to the g-2 experiment[6]. This FEB system will be used for the extraction of the $28 \mathrm{GeV}$ high intensity proton beam which will irradiate the "neutrino production" target. A section of the AtR beam transfer line, which is used to transfer the extracted beam from AGS into the Relativistic Heavy Ion Collider (RHIC), will also be used to transport the $28 \mathrm{GeV}$ proton beam to the "neutrino production" target [2]. Description of the FEB system has been written in ref. $[4,6]$.

\subsection{Layout of the AGS Fast Extracted Beam (FEB)}

A schematic diagram of the Fast Extracted Beam (FEB) in AGS is shown in Fig. 6. During beam extraction the circulating beam is "locally bumped" at two locations along the AGS ring, one location is the fast extraction kicker ( FK ) and the other location is the septum magnet (H10) both shown in Fig. 6. The bunched beam is then extracted (dotted line in fig 6) upon the firing of the fast extraction kicker (FK) shown in Fig. 6. Finally the H10 septum magnet extracts the beam into the AtR transfer line.

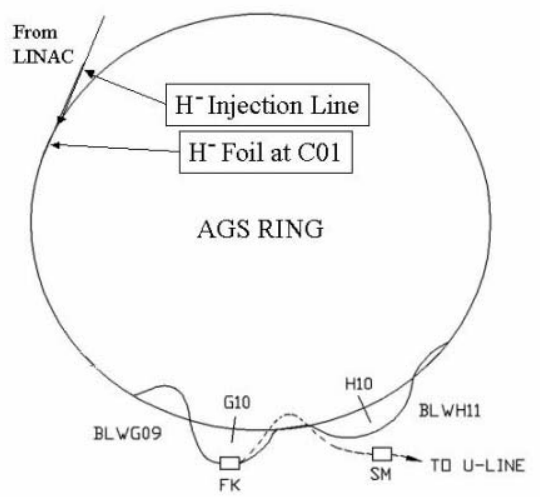

Figure 6. Schematic diagram of the Fast Extracted Beam in AGS.

\subsection{Beam Parameters at the AGS Extraction Point}

The beam transport line to the neutrino target is discussed in ref [2]. The beam parameters which are used in the optics of the transport line from the AGS to the neutrino production target have been calculated [4] and measured experimentally[7] and appear in Table 2.

TABLE 2: Theoretical (Theo.) and measured (Meas.) beam parameters at the "Extraction point" of the AGS.

\begin{tabular}{|l|c|c|c|c|c|c|}
\hline Loc. & $\beta_{\mathrm{x}} \mathrm{m}$ & $\alpha_{\mathrm{x}}$ & $\eta_{\mathrm{x}} \mathrm{m}$ & $\eta^{\prime}{ }_{\mathrm{x}}$ & $\beta_{\mathrm{y}} \mathrm{m}$ & $\alpha_{\mathrm{y}}$ \\
\hline EXT. Theo. & 37.5 & -4.1 & -1.5 & -0.13 & 6.5 & 0.85 \\
\hline EXT. Meas. & 36.7 & -4.5 & & & 7.7 & 1.2 \\
\hline
\end{tabular}

\section{CONCLUSIONS}

The results of the present study show that it is feasible to inject an $\mathrm{H}^{-}$beam at $1.2 \mathrm{GeV}$ into AGS at a frequency of $2.5 \mathrm{~Hz}$, accelerate it to $28 \mathrm{GeV}$ and extract it to the neutrino production target.

\section{REFERENCES}

[1] "Report on the BNL Neutrino Working Group" BNL69395 Informal Report Oct. 2002

[2] BNL-71228 Informal Report

[3] A. Ruggiero et. al. These Proceedings

[4] N. Tsoupas, et. al. "Closed Orbit Calculations in AGS and Beam Parameters at Extraction" AD/RHIC/RD-75

[5] D.S. Barton "Charge Exchange Injection at the AGS" BNL Int. Rep. 32784 PAC, Santa Fe NM, March 31983

[6] N. Tsoupas et. al. "Fast Extracted Beam (FEB) for the g-2 Experiment" CA/AP/54

[7] N. Tsoupas et. al. "A computer code to measure the Beam Emittance and Beam Parameters of the AtR Line" C-A/AP/42 